\title{
ELECTRON SPECTROSCOPIC STUDIES OF THE NEUTTRALIZATION OF SLOW MULTICHARGED IONS DURING INIERACTIONS WITH A METAL SURFACE
}

\author{
F.W. Meyer, L. Folkerts*, C.C. Havener, I.G. Hughes*, S.H. Overbury, D.M. Zehner, \\ and P.A. Zeijlmans van Emmichoven ${ }^{\dagger}$ \\ Oak Ridge National Laboratory, Oak Ridge, Tennessee USA 37831-6362
}

\begin{abstract}
Recent experimental results of electron spectroscopic studies of the neitralization and relaxation of slow multicharged ions interacting with metal surfaces obtained at Oak Ridge National Laboratory are summarized. Discussed are measurements of projectile K-Auger electron emission during interactions of $\mathrm{N}^{6+}$ ions with clean and cesiated $\mathrm{Au}$, as well as clean Cu single crystal targets. While the dominant component in the measured K-Auger spectra is shown to be due to sub-surface electron emission, at incident perpendicular velocities below about $10^{-2}$ au, small features become evident in the measured K-Auger spectra that are consistent with abovesurface projectile electron emission. The experimental results are compared with results of modelling studies of above- and sub-surface electron emission. Also presented are measurements of low energy electron emission during multicharged ion-surface interactions, together with a discussion and analysis of the various contributing low energy emission mechanisms.
\end{abstract}

\section{INTRODUCTION}

The neutralization and relaxation of slow multicharged ions during interactions with surfaces has been the focus of numerous recent studies. A variety of experimental approaclies have been used, ranging from total electron yield ${ }^{1}$ and electron spectrcscopic ${ }^{2-6}$ measurements, to analysis of scattered ions, ${ }^{7}$ and low and high resolution $x$-ray spectroscopy ${ }^{8-10}$. Although still evolving, a consistent picture is beginning to emerge of the overall scenario by which the neutralization and relaxation is thought to occur. It is by now generally accepted that neutralization of multicharged ions can already start well above the surface by classical overbarrier transitions of target valence band electrons to high lying Rydberg levels of the approaching projectile. The critical distance above the surface at which such transitions first become possible is given (in atomic units) by"

$$
R_{c}(W) \sim \sqrt{8 q+2} / 2 W
$$

where $W$ is the surface workfunction of the target material and $q$ is the initial charge state of the incident projectile. The principal quantum number, $n_{c}$ into which the valence electrons are initially captured scales ${ }^{11}$ as $W^{1 / 2}$. For $\mathrm{N}^{6+}$ ions incident on clean $\mathrm{Au}, \mathrm{R}_{\mathrm{c}}$ and $\mathrm{n}_{\mathrm{c}}$ are approximately 20 a.u. and 7 , respectively. Due to the long time scales required for at least two electrons to cascade down to the projectile L-shell, contributions of above-surface KLL-Auger transitions to the total observed projectile K-Auger electron emission are seen only at very low incident perpendicular velocities.
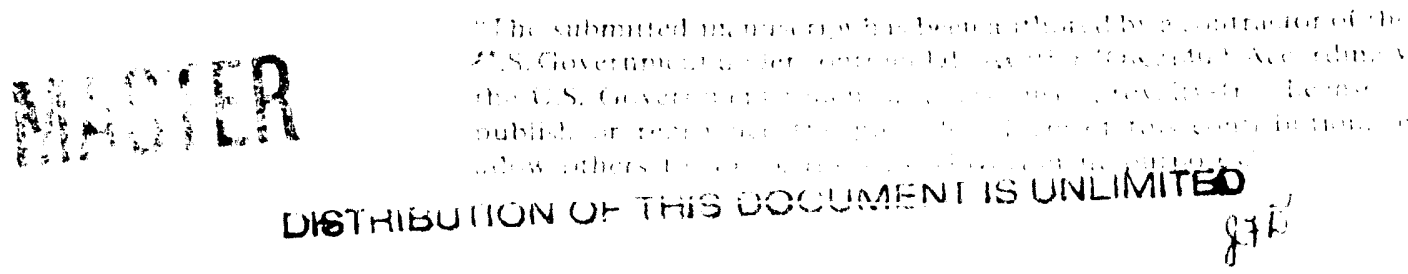
Projectile image-force-acceleration imposes a limit ${ }^{1,5,11}$ on the lowest perpendicular velocity achievable, with the consequence that the observed above-surface K-Auger yield is limited to less than about $20 \%$ of the total K-Auger emission ${ }^{15}$. After penetration of the surface, screening effects facilitate direct capture into lower projectile $n$-levels (for $\mathrm{N}^{6+}$ projectiles, $n=2-3$ ), leading to a very fast filling of the surviving $\mathrm{K}$-shell vacancies. The total $\mathrm{K}$-Auger electron emission thus arises predominantly as a result of sub-surface processes.

In this progress report, recent experimental results of studies on low energy multicharged ion surface interactions obtained at Oak Ridge National Laboratory are summarized. Comparisons of the measurement results and results of modelling studies of above- and sub-surface electron emission are presented and discussed within the framework of the above-outlined scenario.

\section{EXPERIMENTAL APPROACH}

Most of the details of the experimental apparatus and procedure have been previously described". Briefly, an ion beam of selected energy extracted from the ORNL ECR ion source is magnetically analyzed and then collimated by passage through two small apertures to give a 1 -mm-diam spot size on a clean single-crystal metal target at normal incidence. Prior to multicharged-ion-induced electronemission measurements the metal targets were sputter cleaned using $1 \mathrm{keV}$ $\mathrm{Ar}^{+}$ions; surface cleanliness was verified using electron-induced Auger spectroscopy. The angle of incidence of the ion beam, which determines the perpendicular approach velocity, could be varied in the entire range between normal and extreme grazing conditions. The perpendicular ion approach velocity could, of course, also be varied by changing the total ion kinetic energy at fixed incidence angle. However, in this case, it is more difficult to compare spectral features at different perpendicular velocities, since strongly energydependent collisional broadening effects and different sub-surface neutralization dynamics alter the sub-surface projectile K-Auger peak shape.

Electron spectra were measured at selected angles relative to the beam direction using a small spherical sector energy analyzer with a nominal energy resolution of $2.8 \%$. As part of the normalization procedure used to place the spectra on an absolute scale, a

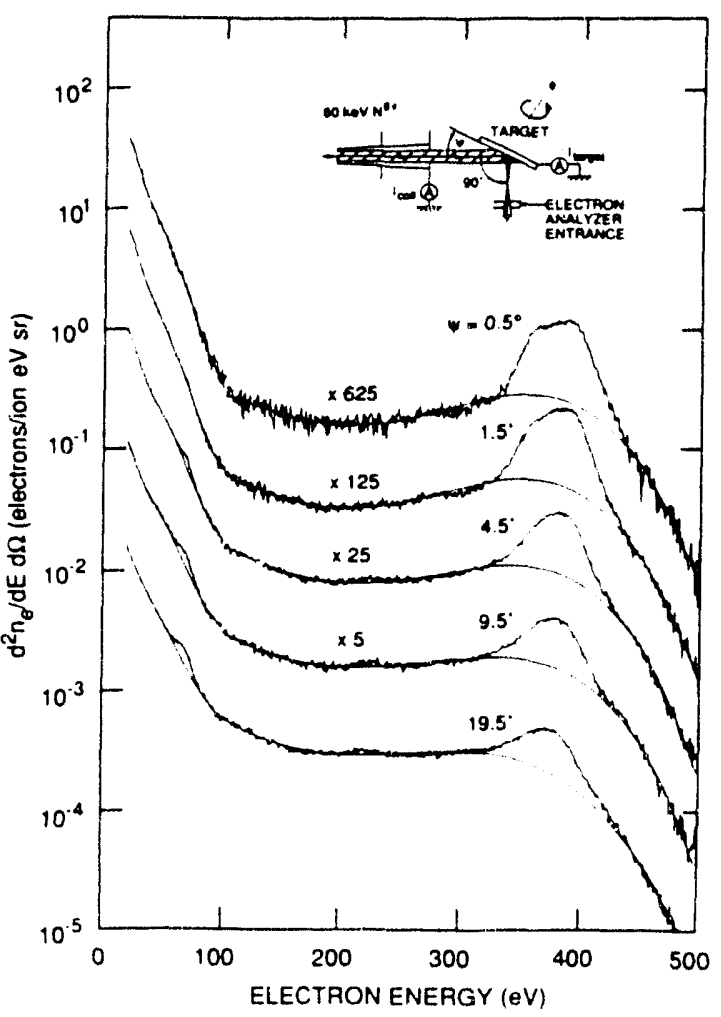

Fig. 1. Electron spectra for $60 \mathrm{keV} \mathrm{N}^{6+}$ incident on $A u(001)$ for various incidence angles. 
geometric correction factor was applied for angles of incidence less than about $15^{\circ}$, when the target region illuminated by the incident beam was larger than the experimentally determined 3.3-mm-diam viewing region of the electron spectrometer. For incidence angles below $5^{\circ}$ the effective beam divergence was less than $\pm 0.2^{\circ}$. Figure 1 shows typical electron spectra acquired for $60 \mathrm{keV} \mathrm{N}^{6+}$ ions incident on clean $A u(001)$ for incidence angles in the range $0.5-19.5^{\circ}$; the inset of the figure gives details of the experimental configuration used.

For reliable quantitative measurements at extreme grazing angles, an accurate calibration $^{12}$ of the incidence angle of the ion beam on the metal target is required. This was accomplished through an auxiliary measurement in which the K-Auger electron signal observed perpendicular to the incident beam direction was measured as a function of target manipulator rotation angle. For incidence angles sufficiently small (e.g. for $\mathrm{Cu},<3.4^{\circ}$ ) that the total normalized K-Auger yield has reached its maximum value ${ }^{4}$ (corresponding to one $\mathrm{K}$-Auger decay per incident ion), the decrease in raw K-Auger signal with further decreasing incidence angle is solely due to the decreasing fraction of the incident beam illuminating the target region viewed by the spectrometer, which vanishes at zero incidence angle. As shown in Ref. 12 , extrapolation of the raw K-Auger signal to zero provides an accurate zero incidence angle calibration, while the width of the transition region around "zero" provides a measure of the divergence of the incident beam. It is noted that this technique can also provide a guide for reproducing a given grazing incidence condition subsequent to target sputter cleaning, which requires target rotation away from the orientation used for the grazing incidence measurements.

\section{PROJECTILE K-AUGER EMISSION: CLEAN CU(001) RESULTS}

Figure 2a shows normalized K-Auger electron spectra measured for incidence angles in the range $0.2-2.4^{\circ}$, after background subtraction using a procedure out-

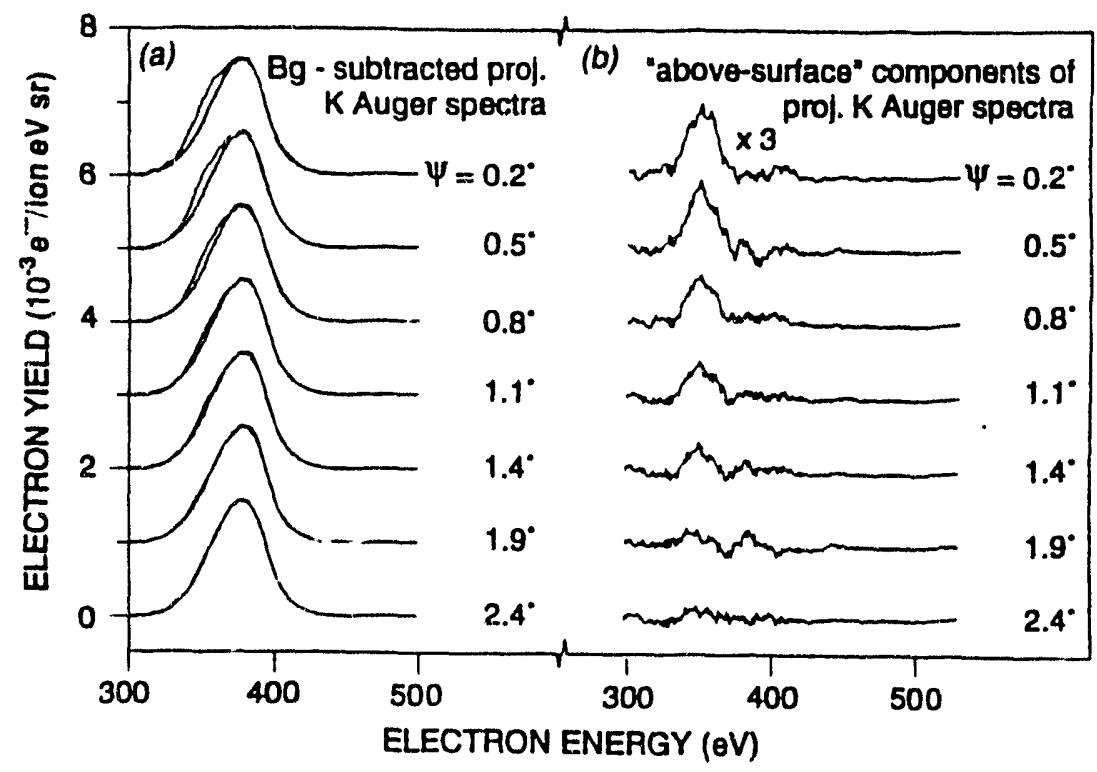

Fig. 2. Background subtracted projectile K-Auger spectra at extreme grazing incidence angles for $60 \mathrm{keV} \mathrm{N}{ }^{6+}$ ions on $\mathrm{Cu}(011)$, with superimposed scaled $7.4^{\circ}$ reference spectra (a), and (b) after subtraction of same [see text]. 
lined in detail in Ref. 4. In this range of incidence angles, a noticeable evolution of the $K$-Auger peak shape occurs, as a progressively more intense component peaked at about $350 \mathrm{eV}$ is seen to appear in addition to the main sub-surface ${ }^{4} \mathrm{~K}$-Auger lineshape peaked at abo ' $380 \mathrm{eV}$. Prior to the appearance of this additional component, i.e. at incidence angles larger than $2.4^{\circ}$, the shapes of the background-subtracted spectra were found to remain remarkably constant, the only variation being in the overall intensity ${ }^{4}$. The peak energy of the additional component is consistent with a minimum L-shell population at the time of the K-Auger decay, as is expected from the slow above-surface autoionization cascade. This "above-surface" component is shown in Fig. $2 b$ as a difference between a particular grazing angle spectrum and a representative large incidence angle $\left(\Psi=7.4^{\circ}\right)$ spectrum that has been superimposed on it in Fig. 2a, after scaling to have the same intensity at $380 \mathrm{eV}$ (where only subsurface emission contributes) as the grazing angle spectrum.

The inverse perpendicular velocity dependence of the intensities of both $\mathrm{K}$ Auger components is summarized in Fig. 3, where the integrated $K$ Auger peak areas are shown for incidence angles up to $20^{\circ}$ (corresponding to the minimum investigated inverse perpendicular velocity of 7 a.u. ${ }^{-1}$ ). The open and solid symbols refer to measurements first published in Refs. 4 and 12 , respectively. Similar results have also been obtained for a Au(011) single crystal target.

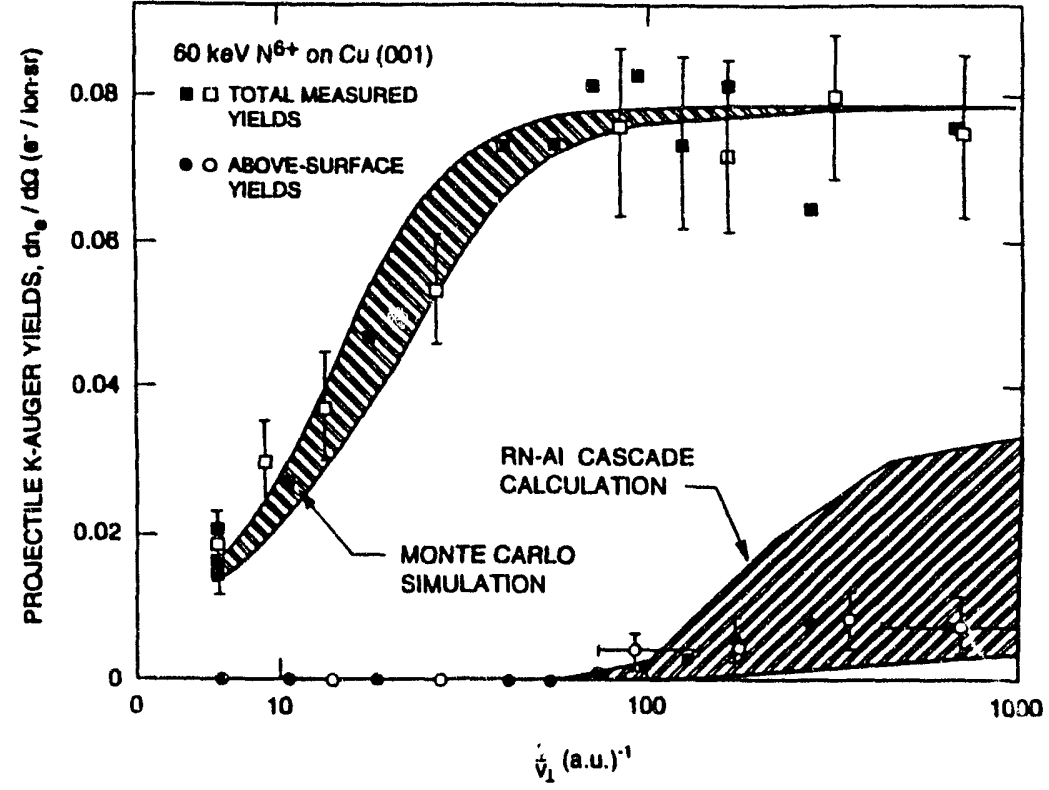

Fig. 3. Above- and sub-surface $\mathrm{K}$-Auger yields (squares and circles, resp.) for $60 \mathrm{keV} \mathrm{N} \mathrm{N}^{6+}$ incident on $\mathrm{Cu}(011)$ vs. $\mathrm{v}_{\perp}^{-1}$, together with simulation results for same.

\section{The identification of the}

main component of the $p_{1}$ jectile K-Auger peak as sub-surface emission is supported by the observation of significant target azimuth dependence found for the intensity of this component, as illustrated in Fig. 4. The experimentally observed target azimuth dependence as well as the $v_{L}^{-1}$ df jendence is in excellent accord with a Monte Carlo simulation of the sub-surface K-Auger emission, also shown in Figs. 3 and 4 . The simulation, described in detail in Refs. 4 and 13, assumes a two step mechanism in the sub-surface K-Auger emission, the first consisting of the filling of the L-shell, described by an adjustable rate $R_{L}$, and the second the KLL transition itself whose rate $R_{K}$ is known. The time evolution of the resulting three component system is solved along actual ion trajectories calculated using the MARLOWE code ${ }^{14}$. Transport of the emitted Auger electron to the target surface is treated using tabulated election inelastic mean free paths. The band delimiting the sub-surface simulation results in 
Fig. 3 represents the variation in simulated K-Auger yields obtained within the $5^{\circ}$ uncertainty range about the nominal [110] mechanical azimuth setting.

Also shown in Fig. 3 are the above-surface $\mathrm{K}$ Auger peak areas. As can be seen. there is a clear saturation ${ }^{4,12}$ of the abovesurface K-Auger electron emission at small perpendicular velocities, as also observed by Das and Morgenstern', which is ascribed to projectile image-potential acceleration. In addition, the results show a threshold of abovesurface K-Auger electron emission in the inverse

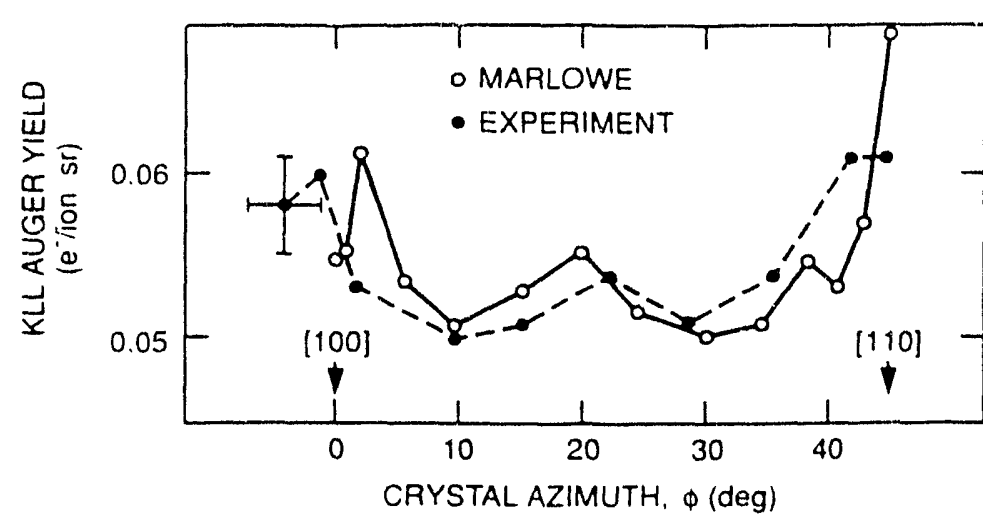

Fig. 4. Comparison of simulated and measured $\mathrm{Cu}$ target azimuth dependence of K-Auger electron emission for $60 \mathrm{keV} \mathrm{N}{ }^{6+}$ incident on $\mathrm{Cu}(001)$ at $5 .^{\circ}$ perpendicularvelocity inter-

val $57-73$ a.u..$^{-1}$ (incidence angle range $2.4^{\circ}>\Psi>1.9^{\circ}$ ), in excellent agreement with classical over-the-barrier simulation ${ }^{11}$ results for the above-surface resonant-neutralization/autoionization (RN-Al) cascade, also shown in Fig. 3. This calculation features a self-consistent and dynamical treatment of the resonance neutralization process and the projectile effective charge, that allows for multiple electron capture as well as loss. The calculation explicitly includes the effect of the dynamical image potential both on the projectile energy levels as well as on the projectile trajectories. Image-potentialacceleration of the projectile is thus accounted for. The indicated band of results shown in Fig. 3 represents the variation of results obtained under different assumptions for the forms of the image potentials and screening functions, as well as differen 8 choices of the Auger rates (see Ref. 11 for additional details).

\section{PROJECTILE K-AUGER EMISSION: CESIATED AU(110) SURFACE RESULTS}

To determine the effect of surface-workfunction-reduction on the above-surface neutralization dynamics, measurements of projectile K-Auger electron emission were performed using a cesiated Au surface ${ }^{15}$. To facilitate these measurements, a Cs dispenser with shutter was mounted in our experimental chamber approximately $6 \mathrm{~cm}$ from the single crystal Au target. By resistive heating of the dispenser above a threshold temperature monitored by a chromel-alumel thermocouple, a reproducible flux of Cs could be obtained to which the Au crystal could be exposed. Using a retarding field method with normally incident $200 \mathrm{eV}$ electrons, the surface work function was found to decrease in proportion to Cs dosing time up to about $0.5 \mathrm{Cs}$ monolayer coverage, corresponding to a work function decrease of approximately $3 \mathrm{eV}$.

Using the above procedure, projectile K-Auger spectra were measured at incidence angles in the range $0.3-5^{\circ}$ for clean and cesiated $\mathrm{Au}$. The results are illustrated in Fig. 5. For the $1.4^{\circ}$ and $0.7^{\circ}$ incidence measurements (top two panels of Fig. 5), the surface was first prepared by Cs dosing and the change in work 
function (relative to that for clean $A(:)$ measured as described above. In situ thermal desorption was then employed to remove the Cs overlayer, after which another electron spectrum was acquired (right half of each panel). Subsequerit to this measurement the loss of Cs was confirmed by a second work function determination. The only difference in experimental conditions between the two spectra was thus the amount of Cs coverage. While significantly reducing the Cs coverage in each case (see measured work function changes noted in each panel), the thermal desorption cycles were unsuccessful in completely removing the Cs overlayer. For this reason, the spectra for the smallest incidence angle were measured in reverse order, starting with a freshly sputter cleaned sample.

As has been previously verified both for $\mathrm{Au}$ and $\mathrm{Cu}$ targets ${ }^{4,12}$, at an incidence angle of $5^{\circ}$ the K-Auger peak arises solely from sub-surface neutrali-

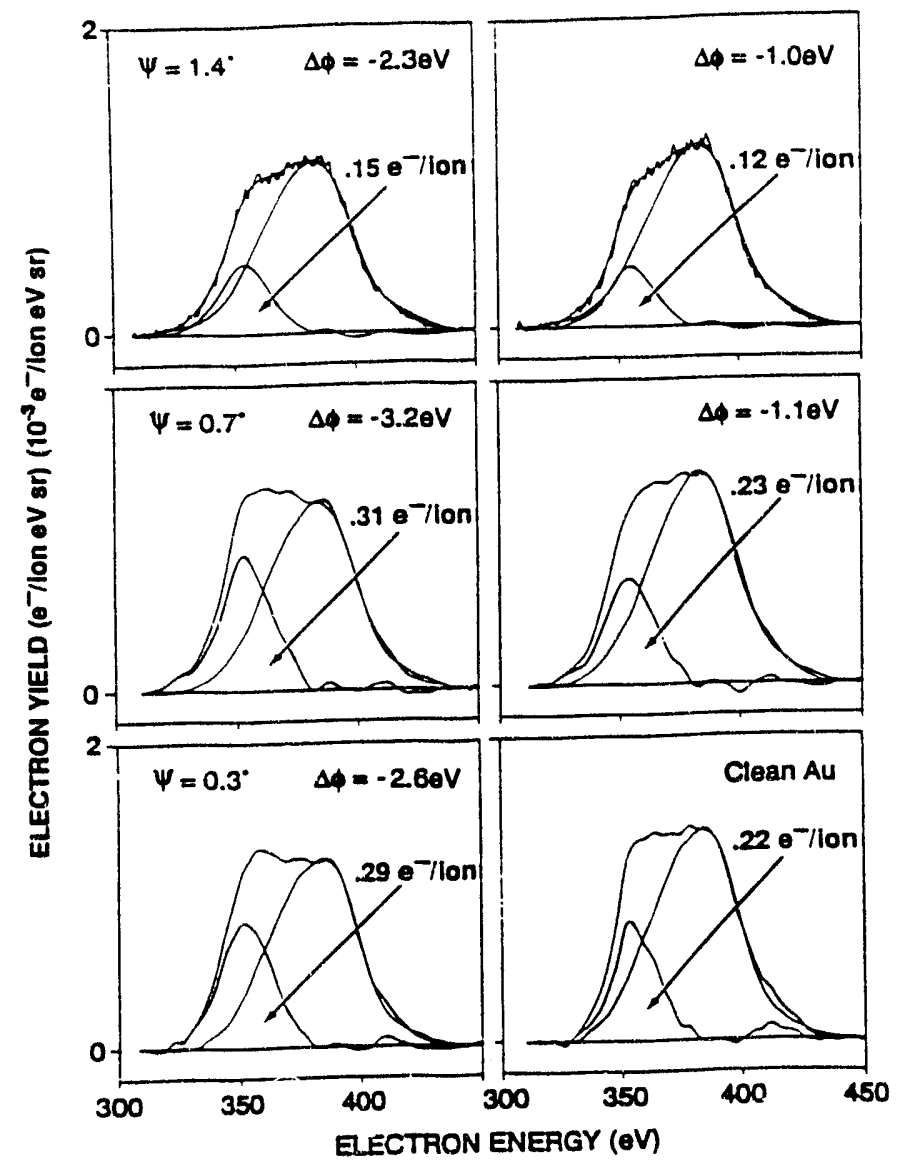

Fig. 5. Background subtracted projectile K-Auger peaks for $60 \mathrm{keV} \mathrm{N}^{6+}$ grazingly incident on cesiated and clean $A u(110)$, together with $5^{\circ}$ reference spectra used to estimate the sub-surface components.

zation and relaxation processes.

In the present measurements, the shapes of the $5^{\circ} \mathrm{K}$-Auger peaks, also shown in Fig. 5, were found to be independent of Cs coverage in the $0-0.7$ monolayer range investigated, and were used as estimates of the sub-surface components in the more grazing incidence angle spectra in a manner already described in the previous section. As can be seen from the spectra shown on the right in Fig. 5, pronounced above-surface components are evident in all the grazing incidence spectra even in the case of minimal Cs coverage. Furthermore, significant enhancements of these abovesurface K-Auger components were observed for the reduced work function surfaces produced by Cs deposition (spectra on left in Fig. 5).

From Eq.(1) it can be seen that, for the work function reductions indicated in Fig. 5, more than a factor of two increase in the above-surface critical distance should have been realized. With neutralization starting at larger distances above the surface, the image potential acceleration of the projectile will likely be significantly reduced, which should increase the time available for the above-surface cescade and subsequent K-Auger emission, and therefore increase the observed above-surface KAuger yield. On the other hand, the increase in the critical distance for first electron 
capture by the projectile is accompanied by an increase in the principal quantum number initially populated, due to the reduced binding energy of the neutralizing target (i.e., Cs) electrons, and the reduced upward projectile energy level shifts due to the image potential interaction at the larger above-surface distances. It is therefore to be expected that the subsequent deexcitation cascade populating the projectile inner shells will require more time. In addition, certainly for a thick Cs layer, the surface electron density of states at the Fermi level may be significantly reduced relative to the clean $\mathrm{Au}$ case, thereby reducing the number of metal electrons available for projectile neutralization. The latter two effects would tend to decrease the above-surface K-Auger electron emission for a particular interaction time. In light of these competing factors, it is interesting to note that an overall enhancement of the above-surface K-Auger yield, approaching $35 \%$ at some of the incidence angles, was observed.

The experimentally determined above-surface K-Auger electron emission is shown in Fig. 6, together with simulation results from the previously described over-the-barrier above-surface neutralization code, modified $^{15}$ to permit treatment of a cesiated surface. The figure shows the inverse perpendicular velocity dependence of the simulated $\mathrm{K}$-Auger yields for a number of different surface workfunctions in the range 0.1-0.187 a.u. The other simulation parameters were kept fixed to reproduce approximately the "upper bound" simulation results for clean $\mathrm{Au}$ (see Ref. 4). The dominant effect of the work

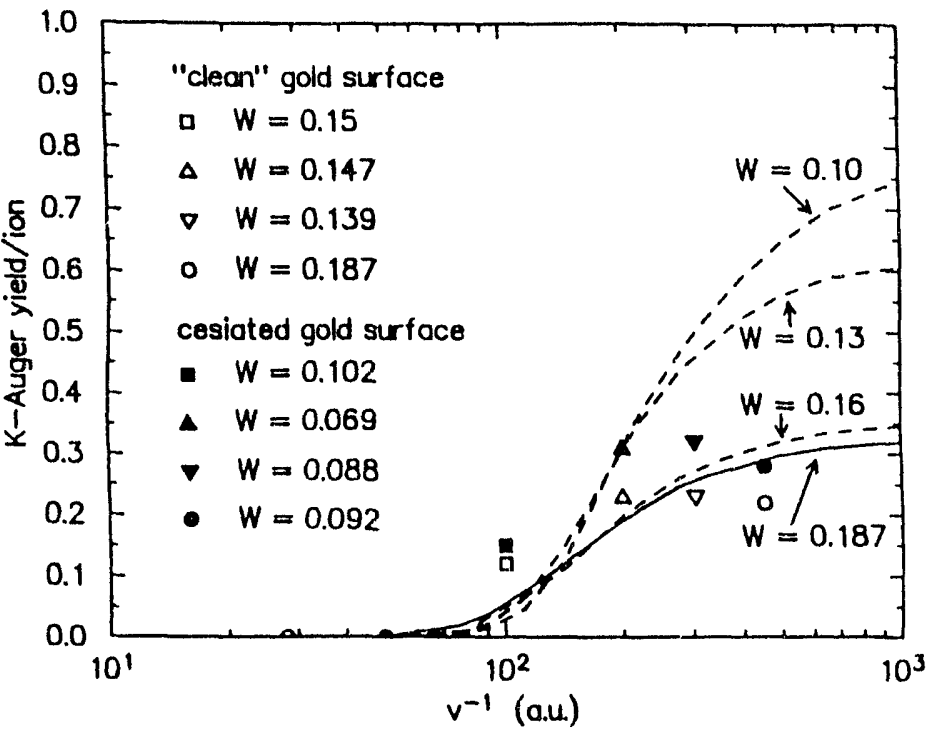

Fig. 6. $v^{-1}$ dependence of the simulated abovesurface K-Auger yields for $\mathrm{N}^{6+}$ incident on clean and cesiated $\mathrm{Au}$, for range of surface work function values compared with experimental results. function reduction on the simulated above-surface yields is found ${ }^{15}$ to be ine suppression of the image acceleration for the ion in front of the cesiaked surface at large distances due to the screening by the charge cloud of electrons already captured in outer shells. The increased interaction time available for the Auger cascade resulting from the increased critical distance is found to be largely offset by slower Auger rates in the higher n-shells. While reasonable agreement is found between the simulation results and the experimentally deduced above-surface $\mathrm{K}$-Auger yields at perpendicular velocities a.bove $10^{-2}$ a.u., at lower velocities the simulation appears to significantly overestimate the observed above-surface K-Auger yield enhancement. The reasons for this discrepancy are at present not completely understood. 


\section{LOW ENERGY ELECTRON EMISSION}

The results shown in the preceding sections have suggested that, even at the lowest incident perpendicular velocities, only a small fraction of incident ions carrying deep lying inner shell vacancies will be completely neutralized and relaxed prior to impact on the target surface, the majority still being in a variety of multiexcited states. Upon surface penetration the sudden screening of the projectile core charge by target valence band electrons will result in any electrons bound to the projectile in shells $n \geq 4$ becoming unbound and "peeled" from the projectile. Those peeloff electrons that escape into the vacuum are expected to have energies less than 50 $\mathrm{eV}$. Low energy electrons are also expected

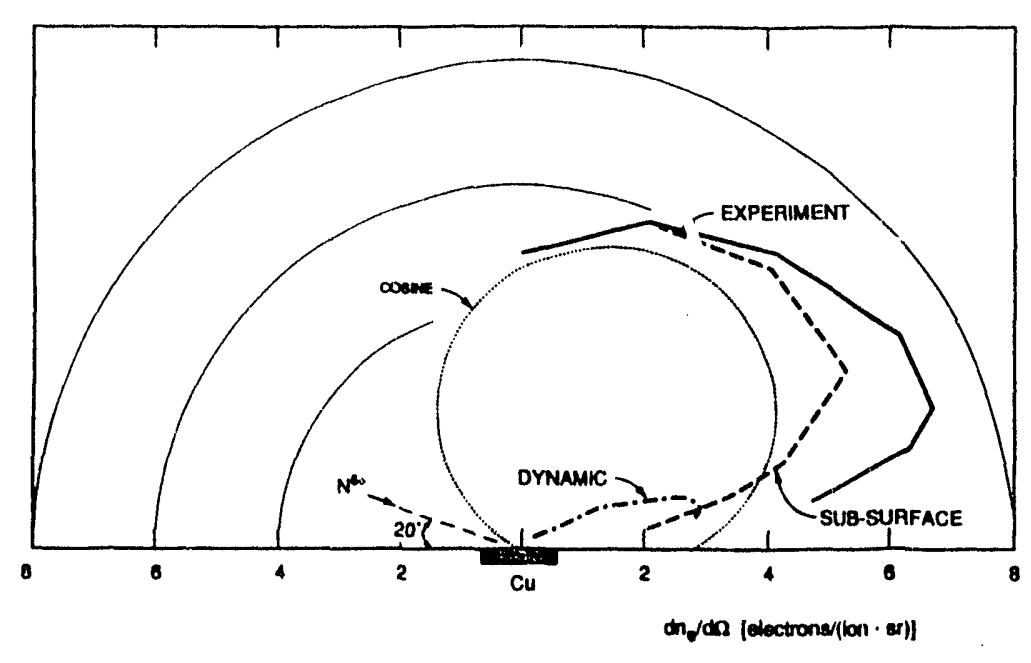

Fig. 7. Polar plot of the angular dependence of the energyintegrated electron emission for $100 \mathrm{keV} \mathrm{N}^{6+}-\mathrm{Cu}(001)$ collisions, showing "sub-surface" and "dynamic" components. to be produced during the early stages of the above-surface autoionization cascade involving shells $n \geq 3$, as well as during above-surface promotion into the continuum of projectile Rydberg electrons due to their interaction with the projectile image potential or due to screening effects. At our investigated projectile energies, there is the additional large contribution of low energy kinetically emitted electrons which complicates the evaluation of the relative contributions of the above potential emission processes to the total electron yield.

We have made measurements of energy and angle resolved low energy electron emission ${ }^{16}$ as well as total electron yields ${ }^{17}$ during multicharged ion interactions with $\mathrm{Au}$ and $\mathrm{Cu}$ surfaces. As is illustrated in Fig. 7, the low energy electron emission was found to consist of a dominant "sub-surface" component characterized by $\operatorname{a} \cos (\theta)$-like angular distribution skewed in the forward direction, and a much smaller "dynamic" component, possibly created during binary encounters with metal electrons at the surface, which is strongly peaked at extreme forward angles.

It was found in addition that both the emission at a particular analyzed electron energy as well as the total electron yields exhibit characteristic variations in magnitude with target azimuthal orientation relative to the incident multicharged ion beam. This is illustrated in Fig. 8 for electron emission at $20 \mathrm{eV}$ for $30 \mathrm{keV} \mathrm{N} \mathrm{N}^{2+}, \mathrm{N}^{5+}$, and $\mathrm{N}^{6+}$ ions incident at $20^{\circ}$ on $\mathrm{Au}(011)$. As can be seen in Fig. 9, we find similar azimuthal variations in the total electron yields. In the velocity range $0.25-0.55$ a.u., the amplitude of the azimuth variations was observed to increase with increasing velocity. 
In the same velocity range, the electron yield differences between different charge states were remarkably target-azimuth, as well as projectile-velocity independent. Calculations of projectile energy loss during traversal of the top $40 \AA$ of the target, made using the MARLOWE code, exhibit the same azimuthal variations, as demonstrated in Fig. 9. We therefore attribute the azimuthally varying component of the eleciron yield to kinetic electron emission. The additional velocity-independent emission for the higher charge states (e.g. $\mathrm{N}^{6+}$ compared with $\mathrm{N}^{5+}$ ) is ascribed to potential emission processes.

Simulation results for the above-surface electron emission processes enumerated at the beginning of this section suggest that, in the investigated velocity regime, the above-surface Auger cascade and promotion to the continuum make only minor contributions to the electron yield. In addition, the maximum possible contribution from peel-off electrons to the yield difference between $\mathrm{N}^{5+}$ and $\mathrm{N}^{6+}$, shown in Fig. 9, is on the order of one electron. The observed yield difference of $3.5 \mathrm{e}$ /ion can thus not be accounted for by above-surface processes alone. It is suggested that a major contribution to the observed yield difference must be due to the presence of the initial K-shell vacancy on the incident $\mathrm{N}^{6+}$ ion. The sub-surface filling of this inner shell vacancy in the first $30 \AA$ of the target gives rise to isotropically emitted $380 \mathrm{eV}$ electrons, which can in turn create low energy secondary electrons. Using published data ${ }^{18}$ of electron
Fig. 8. Target azimuth dependence of $20 \mathrm{eV}$ electron emission for $30 \mathrm{keV} \mathrm{N} \mathrm{N}^{q+}$ incident on $\mathrm{Au}(011)$.

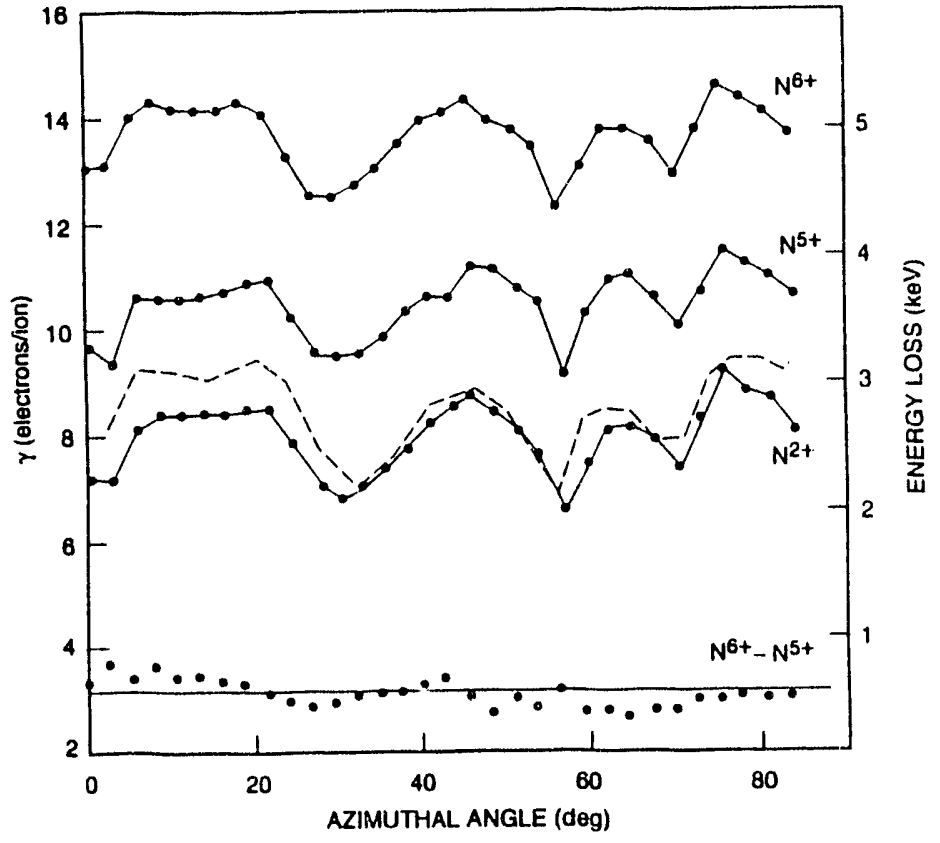

Fig. 9. Target azimuth dependence of $y$ for $30 \mathrm{keV}$ $\mathrm{N}^{q^{+}}$incident on $\mathrm{Au}(011)$ - solid points; dashed line MARLOWE results for projectile energy loss. 
induced secondary electron emission, a rough estimate of $1.5-2 \mathrm{e} /$ ion is obtained. "Indirect" potential electron emission ${ }^{17}$ due to secondary cascade process within the target bulk is thus believed to be a dominant contributor to potential emission in the investigated energy regime.

\section{ACKNOWLEDGEMENTS}

We are indebted to J. Burgdörfer and M.T. Robinson for stimulating discussions and important insights, and to G. Ownby and J. Hale for skilled technical assistance. This work was supported by the Office of Basic Energy Sciences, Division of Chemical Sciences, and the Division of Applied Plasma Physics, Office of Fusion Energy, of the U.S. Department of Energy, under Contract No. DE-AC05-84OR21400 with Martin Marietta Energy Systems, Inc.

*Appointed through the ORAU postdoctoral research program.

tPresent address: Buys Ballotlaboratorium Utrecht, University of Utrecht, NL-3584 CC Utrecht, The Netherlands.

\section{REFERENCES}

'H. Kurz, K. Toglhofer, HP. Winter, F. Aumayr, and R. Mann, Phys. Rev. Lett. 69, 1140 (1992). ${ }^{2} L$. Folkerts and R. Morgenstern, Europhys. Lett. 13, 377 (1990).

${ }^{3}$ H.J. Andră, A. Simionovici, T. Lamy, A. Brenac, G. Lamboley, J.J. Bonet, A. Fleury, M. Bonnefoy, M. Chassevent, S. Andriamonje, and A. Pesnelle, Z. Phys. D 21, S15.

${ }^{4}$ F.W. Meyer, S.H. Overbury, C.C. Havener, P.A. Zeijlmans van Emmichoven, J. Burgdőrfer, and D.M. Zehner, Phys. Rev. A 44, 7214 (1991); F.W. Meyer, S.H. Overbury, C.C. Havener, P.A. Zeijlmans van Emmichoven, and D.M. Zehner, Phys. Rev. Lett. 67, 723 (1991).

5J. Das and R. Morgenstern, Phys. Rev. A 47, R758 (1993).

${ }^{8} \mathrm{~L}$ Folkerts, I.G. Hughes, and F.W. Meyer, Abstracts of Contributed Papers of the XVIII ICPEAC, Aarhus, Denmark.

${ }^{7} \mathrm{H}$. Winter, Europhys. Lett. 18, 207 (1992).

${ }^{8}$ M. Schulz, C. Cocke, S. Hagmann, M. Stőckli, and H. Schmidt-Bőcking, Phys. Rev. A 44, 1653 (1991).

'J.J. Bonnet, A. Fleury, M. Bonnefoy, M. Chassevent, T. Lamy, A. Brenac, A. Simionovici, and H.J. Andrä, Z. Phy's. D Suppl. 21, 343 (1991).

${ }^{10}$ B. d'Etat, J.P. Briand, G. Ban, L de Billy, J.P. Desclaux, and P. Briand, Phys. Rev. A (to be published).

"J. Burgdörfer, P. Lerner, and F.W. Meyer, Phys. Rev. A 44, 5674 (1991); J. Burgdőrfer and F.W. Meyer. Phys. Rev. A 47, R20 (1993).

${ }^{12}$ F.W. Meyer, C.C. Havener, and P.A. Zeijlmans van Emmichoven (submitted to Phys. Rev. A).

${ }^{13}$ S.H. Overbury, F.W. Meyer, and M.T. Robinson, Nucl. Instrum. Methods Phys. Res. B 67, 126 (1992).

${ }^{14}$ M.T. Robinson, Phys. Rev. B27, 5347 (1983); O.S. Oen and M.T. Robinson, Nucl. Instrum. Methods Phys. Res. 132, 647 (1976).

${ }^{15}$ F.W. Meyer, L Folkerts, I.G. Hughes, S.H. Overbury, and J. Burgdőrfer (submitted to Phys. Rev. A).

${ }^{18}$ P.A. Zeijlmans van Emmichoven, C.C. Havener, I.G. Hughes, D.M. Zehner, and F.W. Meyer, Phys. Rev. A47, 3998(1993).

${ }^{17}$ I.G. Hughes, J. Burgdörfer, L. Folkerts, C.C. Havener, S.H. Overbury, M.T. Robinson, D.M. Zehner, P.A. Zeijlmans van Emmichoven, and F.W. Meyer, Phys. Rev. Lett 71, 291 (1993).

${ }^{18}$ S. Thomas and E.B. Pattinson, J. Phys. D 3, 349 (1969). 

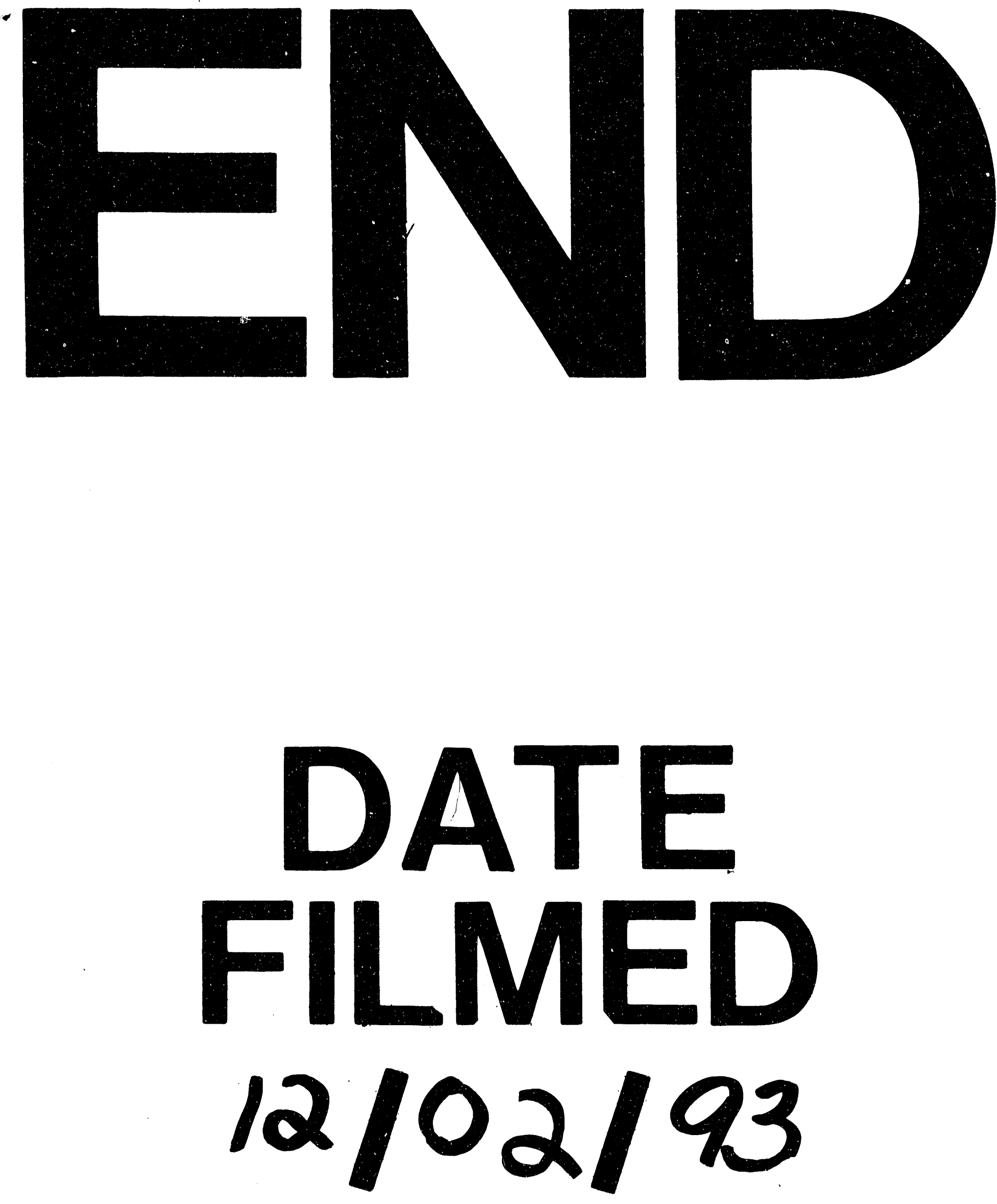

1 
$\ddots$ 